\title{
A numerical simulation of a negative sudden impulse
}

\author{
S. Fujita ${ }^{1}$, T. Tanaka ${ }^{2}$, T. Kikuchi ${ }^{3}$, and S. Tsunomura ${ }^{1}$ \\ ${ }^{1}$ Meteorological College, Kashiwa, Chiba 277-0852, Japan \\ ${ }^{2}$ Department of Geophysics and Planetary Sciences, Kyushu University, Fukuoka 812-8581, Japan \\ ${ }^{3}$ Communications Research Laboratory, Koganei, Tokyo 184-8795, Japan
}

(Received December 22, 2003; Revised April 1, 2004; Accepted April 1, 2004)

\begin{abstract}
A numerical experiment of magnetospheric response to a negative pressure impulse in the solar wind is carried out by using a MHD model of the solar wind-magnetosphere-ionosphere coupled system. The numerical simulation confirms mirror-image relationship of the ionospheric and magnetospheric signatures between the negative and positive impulses, which has been suggested by previous observations. The plasma processes associated with the negative impulse are again divided into the three phases - the preliminary impulse phase, and the first and second main impulse phases in terms of the ionosphere-magnetosphere coupling. The SC transient cell convection in the second main impulse phase is related to the Region 2 current in the case of the negative impulse. In the last, we discuss a possible model for the auroral brightening at the onset of the negative impulse.
\end{abstract}

Key words: Negative SI, numerical simulation, magnetosphere-ionosphere coupling, connection, current system, solar wind impulse.

\section{Introduction}

It is well known that a sudden increase in dynamic pressure of the solar wind invokes a geomagnetic sudden commencement (SC) or a geomagnetic sudden impulse (SI) (e.g., Araki, 1994 and references therein). (When we treat initial response of the magnetosphere to the solar wind impulse, we do not need to discriminate the SC and the SI. Therefore, both are called as the $\mathrm{SI}^{+}$in the present paper.) Geomagnetic variations associated with the $\mathrm{SI}^{+}$are divided into the preliminary impulse (PI) and the main impulse (MI) at the auroral latitudes (Araki, 1994). Besides, geomagnetic variations opposite to those of the $\mathrm{SI}^{+}$are sometimes detected on the ground. This is called as a negative geomagnetic sudden impulse (denoted as the $\mathrm{SI}^{-}$).

The $\mathrm{SI}^{-}$has not been investigated so extensively compared with the $\mathrm{SI}^{+}$. The first systematic study about the $\mathrm{SI}^{-}$was done by Araki and Nagano (1988). They revealed that geomagnetic variations associated with the $\mathrm{SI}^{-}$have directions opposite to those of the $\mathrm{SI}^{+}$both at mid- and lowlatitudes and at high latitudes. Therefore, they concluded that sudden expansion of the magnetosphere yields the $\mathrm{SI}^{-}$ signatures on the ground. Recently, by analyzing plasma data observed in the solar wind and in the magnetosphere associated with the $\mathrm{SI}^{-}$, Takeuchi et al. $(2000,2002)$ confirmed that a sudden decrease in the solar wind dynamic pressure invokes the $\mathrm{SI}^{-}$. They also confirmed mirror-image relation between the $\mathrm{SI}^{-}$and the $\mathrm{SI}^{+}$as discussed by Araki and Nagano (1988). (Only the polarization of the geomagnetic variation is common between the $\mathrm{SI}^{+}$and the $\mathrm{SI}^{-}$as explained by Takeuchi et al. (2000).)

Recently, several numerical works (e.g., Tanaka, 1995, 1999, 2000a; Slinker et al., 1999; Keller et al., 2002; Fujita et al., 2003a, b) revealed that global MHD phenomena are successfully simulated by using a solar wind- magnetosphere-ionosphere model. In addition to realistic reproduction of such phenomena, the simulation (in other words, the numerical experiment) is now regarded as a tool indispensable to investigating physical processes involved in these phenomena. For example, by analyzing numerical results of magnetospheric response to a solar wind pressure pulse, Fujita et al. (2003a, b) manifested plasma processes involved in the $\mathrm{SI}^{+}$. They proposed three phases of the $\mathrm{SI}^{+}$the PI phase, and the first and the second stages of the MI phase. In the PI phase, there appears a counterclockwise current vortex in the magnetospheric equatorial plane; this vortex consists of an enhanced dawn-to-dusk magnetopause current and its partial return current in the inner magnetosphere. The return current is located in the wavefront of the compressional wave launched by sudden compression of the magnetosphere. In the inner magnetosphere, the return current is converted to a field-aligned current (FAC). (This numerical result confirms the theoretical model of the PI presented by Tamao (1964a, b, 1965).) It is noted that the magnetospheric plasma flow does not correspond to the ionospheric flow in the PI phase. On the other hand, in the last phase (the second stage of the MI phase), the magnetospheric convection corresponds to the ionospheric convection. There appears a peculiar convection vortex named by the SC transient cell convection (Fujita et al., 2003b). The current system is essentially the Region 1 current system (Iijima and Potemra, 1976) modified by small-scale pressure enhancement due to compression of the magnetospheric flank. The middle phase (the first stage of the MI phase) is a transition phase between the PI phase and the second stage of the MI phase.

It is noted that no one has attempted to study plasma processes involved in magnetospheric response to a negative solar wind impulse. Therefore, we need to carry out a numerical experiment of the $\mathrm{SI}^{-}$. In our numerical experiment, a 

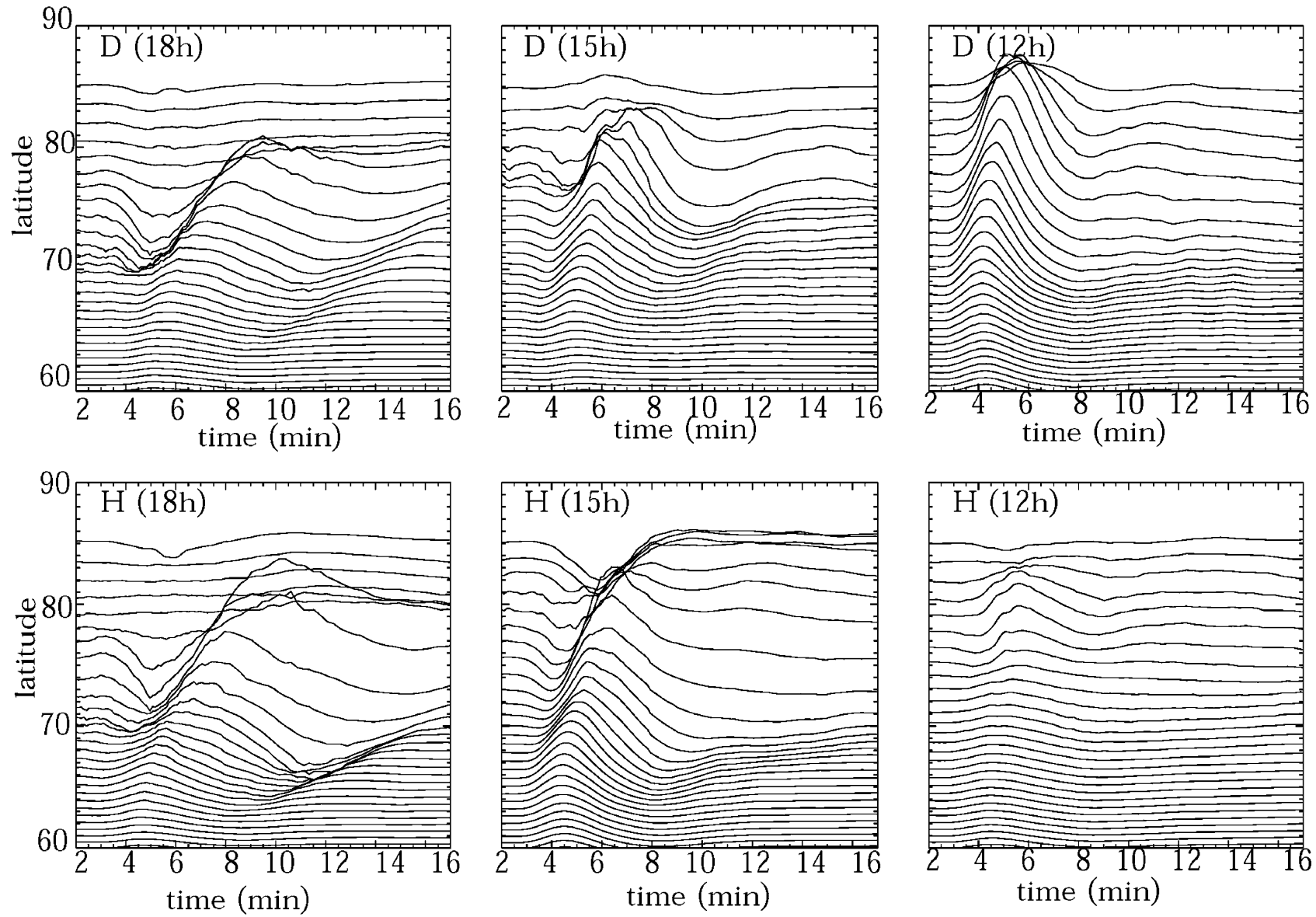

Fig. 1. (Top) Stacked variations of the ground D (east-west) component and (bottom) those of the $\mathrm{H}$ (north-south) component in $60^{\circ}$ to $85^{\circ}$ of latitude from 2 to $17 \mathrm{~min}$. Left, middle, and right panels are at $18 \mathrm{~h}, 15 \mathrm{~h}, 12 \mathrm{~h} \mathrm{LT}$, respectively. The distance between ticks (corresponding to a tick of $1^{\circ}$ ) in vertical axes correspond to $5.6 B_{g 0}$ for $\mathrm{D}$ and $2.8 B_{g 0}$ for $\mathrm{H}$.

negative stepwise impulse of the solar wind density is imposed in the upstream region of the Earth's bow shock. The numerical simulation technique and the model are explained in detail in Section 2.

The present paper is structured as follows. Section 2 explains the numerical technique used for the present MHD simulation and the magnetosphere-ionosphere model. Section 3 describes plasma processes in the $\mathrm{SI}^{-}$event. We mainly discuss relationship between the $\mathrm{SI}^{-}$and the $\mathrm{SI}^{+}$. Section 4 discusses the $\mathrm{SI}^{-}$-triggered auroras. Section 5 summarizes main results.

\section{Numerical Simulation Technique and the Magnetosphere-Ionosphere Model}

A numerical MHD simulation is adopted for the study of the present problem (Tanaka, 1994, 1995, 1999, 2000a, b). The MHD calculation employs a finite volume (FV) totalvariation diminishing (TVD) scheme with an unstructured grid system (Tanaka, 1994), to achieve high resolution and an excellent capture of discontinuities. In the ionosphere, Ohm's law is solved to match the divergence of the Pedersen and Hall currents with the FAC. The ionospheric conductivity is calculated from the solar EUV flux, diffuse precipitation modeled by the pressure and temperature, and discrete precipitation modeled by the upward FAC. In this paper, the
Hall conductivity is set to be two times larger than the Pedersen conductivity. Details for the calculation of conductivity follow Tanaka (2000a). To obtain the ionospheric potential, a two-dimensional partial differential equation is solved on a sphere. The outer boundary conditions give a solar wind flow on the upstream side at $x=40 R_{e}$ and give zero gradient conditions on the downstream side at $x=200 R_{e}$. A uniform solar wind with a speed of $350 \mathrm{~km} / \mathrm{sec}$ and an IMF magnitude of $5 \mathrm{nT}$ is assumed at the upstream boundary. The IMF direction is inclined $30^{\circ}$ from the due northward direction ( $B_{x}=0.0 \mathrm{nT}, B_{y}=2.50 \mathrm{nT}$, and $\left.B_{z}=4.33 \mathrm{nT}\right)$. These solar wind parameters are unchanged throughout the calculation.

The solar impulse is reverse to that used by Fujita et al. (2003a, b). Namely, the plasma density in the solar wind is $25 \mathrm{cc}$ and $10 \mathrm{cc}$, respectively, in the downstream and the upstream of the solar wind impulse front. The time axis is selected so that the solar wind pressure impulse starts in the upstream region of the Earth's bow shock $\left(x=18 R_{e}\right)$ at 0.0 min. The impulse arrives at the magnetopause at about 2 $\min$.

Physical quantities used in this paper are normalized with respect to the following values: plasma flow velocity (denoted as $V_{0}$ in the text) $=53.0 \mathrm{~km} / \mathrm{sec}$; ground magnetic flux density $\left(B_{g 0}\right)=1.0 \mathrm{nT}$; electric current density $\left(J_{0}\right)=$ 

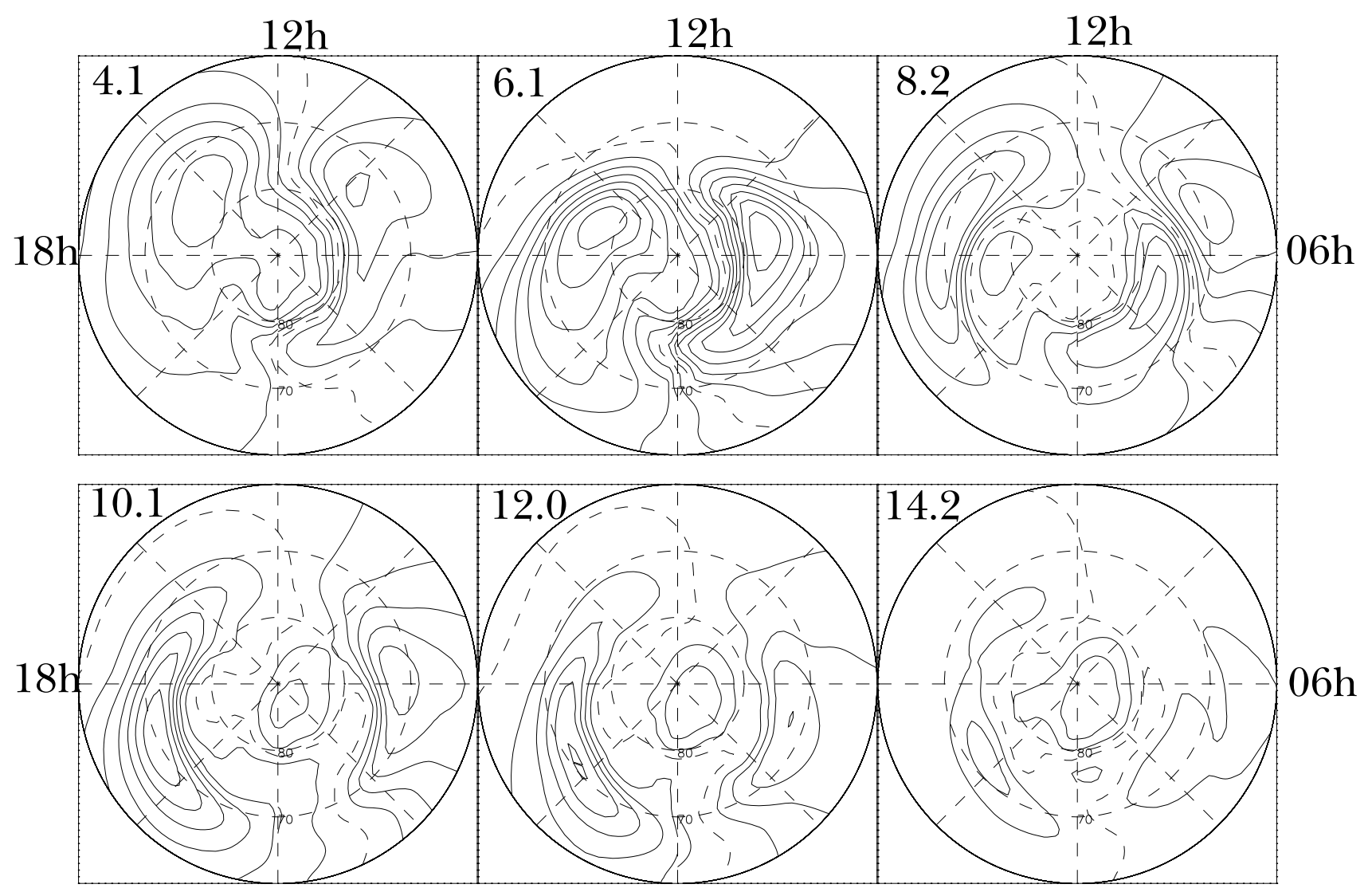

Fig. 2. Equi-contours of ionospheric electric potential at latitudes higher than $60^{\circ}$. The potential is positive (negative) on the thick (thin) contours. The broken contours mean zero potential. The contour interval is $1.5 \phi_{0}$. Number in the figure denotes time in minute.

$0.0125 \mu \mathrm{A} / \mathrm{m}^{2}$; electric field $\left(E_{0}\right)=0.532 \mathrm{mV} / \mathrm{m}$; electric potential $\left(\Phi_{0}\right)=3.39 \mathrm{kV}$; and pressure $\left(p_{0}\right)=46.9 \mathrm{pPa}$.

\section{Plasma Processes Associated with a Simulated $\mathrm{SI}^{-}$}

This section presents ground magnetic variations and ionospheric disturbances associated with a negative solar wind impulse impinging on the magnetosphere. After showing correspondence between simulated ground magnetic variations and observed geomagnetic signatures of the $\mathrm{SI}^{-}$, we present ionospheric electric potential and FAC profile in order to clarify characteristic features of the simulated results. In particular, we discuss mirror-image relationship between the $\mathrm{SI}^{-}$and the $\mathrm{SI}^{+}$. In the last part of this section, the magnetospheric current systems and magnetospheric plasma flows will be investigated.

\subsection{Simulated ground magnetic signatures}

Before analyzing the numerical results, we need to confirm that the present simulation reproduces real phenomena correctly. The Biot-Savart's law is utilized in calculation of ground magnetic fields with simplifications that ionospheric currents directly overhead mainly contribute to magnetic fields. In the calculation, we assume that the Hall current produces the ground magnetic field. (The Hall current is calculated as a product of the ionospheric Hall conductivity given in the present model (Tanaka, 1995) and ionospheric electric fields.) This procedure is the same as that used in calculation of the equivalent ionospheric current from the ob- served ground magnetic field.

Figure 1 shows latitudinal profiles of ground magnetic variations associated with the negative solar wind impulse at three local times ( $12 \mathrm{~h}, 15 \mathrm{~h}$, and $18 \mathrm{~h} \mathrm{LT})$. When comparing magnetic variations shown in this figure with the corresponding variations of the $\mathrm{SI}^{+}$shown in figure 4 of Fujita et al. (2003b), we recognize that the simulated variations have mirror-image relation to those of the $\mathrm{SI}^{+}$. For example, the $\mathrm{H}$ component has a negative geomagnetic variation preceded by a positive initial impulsive deflection at the latitudes below $70^{\circ}$ at $15 \mathrm{~h} \mathrm{LT}$. This variation is opposite to the PI variation of the $\mathrm{SI}^{+}$. This mirror-image relationship is consistent with the observation by Araki and Nagano (1988). It is also noted that the simulated $\mathrm{H}$ variation is similar to the magnetogram records observed by the IMAGE magnetometer network at the $\mathrm{SI}^{-}$event (Sato et al., 2001). Finally, it is concluded that the present numerical experiment of a negative solar wind impulse successfully simulates the $\mathrm{SI}^{-}$.

\subsection{Ionospheric signatures of the $\mathrm{SI}^{-}$}

Since ionospheric potential profiles and FAC distributions are essentially anti-symmetric with respect to the noonmidnight meridian, we discuss only the potential and the FAC in the dusk region.

First, we show in Fig. 2 variations of ionospheric electric potentials associated with the $\mathrm{SI}^{-}$at the latitudes higher than $60^{\circ}$ from $4.1 \mathrm{~min}$ to $14.2 \mathrm{~min}$ at about every $2 \mathrm{~min}$. (We do not show the potential before $4 \mathrm{~min}$ because a detectable signature of the $\mathrm{SI}^{-}$appears after $4 \mathrm{~min}$.) In the first two panels 

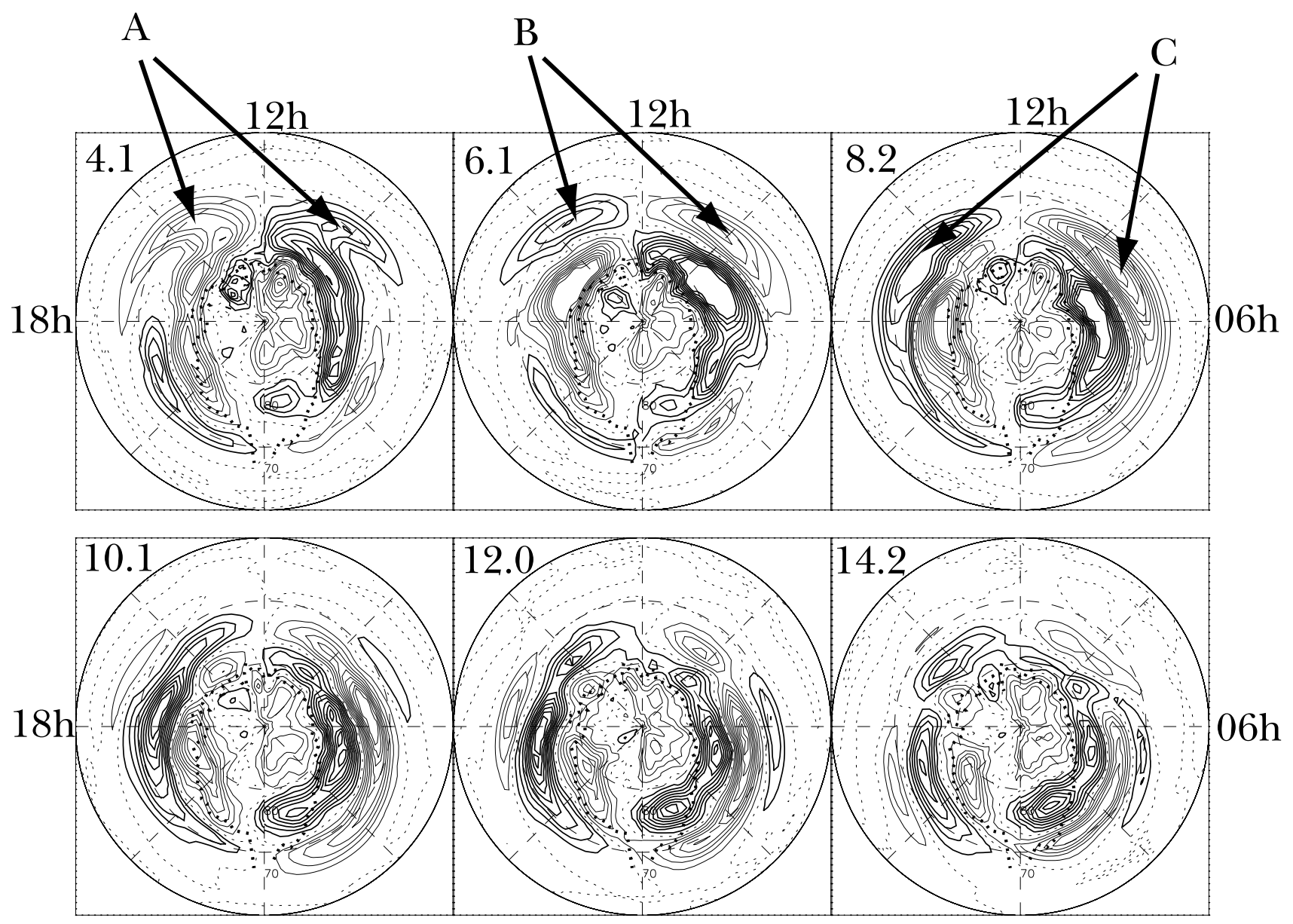

Fig. 3. Equi-contours of ionospheric FAC at latitudes higher than $60^{\circ}$. Thick (thin) contours denote downward (upward) FACs. Dotted contours mean zero FAC. The contour interval is $81 J_{0}$. Dots show the position of the magnetopause. Number in the figure denotes time in minute.

(4.1 $\mathrm{min}$ and $6.1 \mathrm{~min})$, the $\mathrm{SI}^{-}$exhibits a negative potential in the dusk region. This negative potential is replaced by a positive one after $8.2 \mathrm{~min}$. These features are roughly opposite to those for the $\mathrm{SI}^{+}$(figure 3 of Fujita et al., 2003b). Note that the positive potential in the dusk region is most enhanced at $10.1 \mathrm{~min}$. (Temporal variations of the potential and the FAC are discussed later.) Effect of the solar wind impulse disappears after $14.2 \mathrm{~min}$. Then, ionospheric potential distribution becomes close to that in a steady state of the solar wind-magnetosphere-ionosphere system (Tanaka, 1995) in the post-SI ${ }^{-}$period.

Next, Figure 3 shows FAC distributions from $4.1 \mathrm{~min}$ to $14.2 \mathrm{~min}$. In the post-noon region, an upward FAC at the latitudes of about $70^{\circ}$ at $4.1 \mathrm{~min}$ (labeled by "A") is appended to the Region 1 current (the FAC at latitudes of around $80^{\circ}$ ). This additional FAC shifts anti-sunward to be merged into the Region 1 current. At $6.1 \mathrm{~min}$, a downward FAC labeled by "B" appears in the post-noon region at the latitudes of about $70^{\circ}$. This FAC also spreads anti-sunward and is merged into the Region 2 current (the FAC at latitudes of about $70^{\circ}$ ) after $8.2 \mathrm{~min}$. Behavior of the additional FACs (the disturbed part of FACs excited by the solar wind impulse) exhibits basically the mirror-image relationship to that for the $\mathrm{SI}^{+}$(see figure 2 of Fujita et al., 2003b).

Since the additional FACs of the $\mathrm{SI}^{-}$and the $\mathrm{SI}^{+}$appar- ently correspond to each other with opposite directions, it can be regarded that the FACs of "A" and "B" belongs to the PI current system and the fist MI current system, respectively. The second MI current system will be established after about $8 \mathrm{~min}$. Note that, exactly speaking, the current system is discriminated after investigating plasma processes in the magnetosphere. The magnetospheric current system will be treated later.

In the last stage, the FAC pattern essentially consists of the Region $1 / 2$ currents. (We ignore a faint upward/downward FAC in the dusk/dawn side in the region around $70^{\circ}$ because it does not affect significantly the ionospheric potential pattern in Fig. 2.) The Region 2 current is more significant in the second MI phase in contrast to the case of the $\mathrm{SI}^{+}$where the Region 1 current is essential (Fujita et al., 2003b). The peak of the Region 2 current shifts anti-sunward after 8.2 min. This feature will be again discussed.

It is noteworthy that the FACs in the PI phase and in the first stage of the MI phase are divided into the background Region 1/2 currents and additional disturbed part of the FAC in the ionosphere. Therefore, the mirror-image relationship does not hold rigorously for combined FACs (combination of the common Region 1/2 currents and anti-symmetric disturbed FACs) in the ionosphere in the first two phases. 


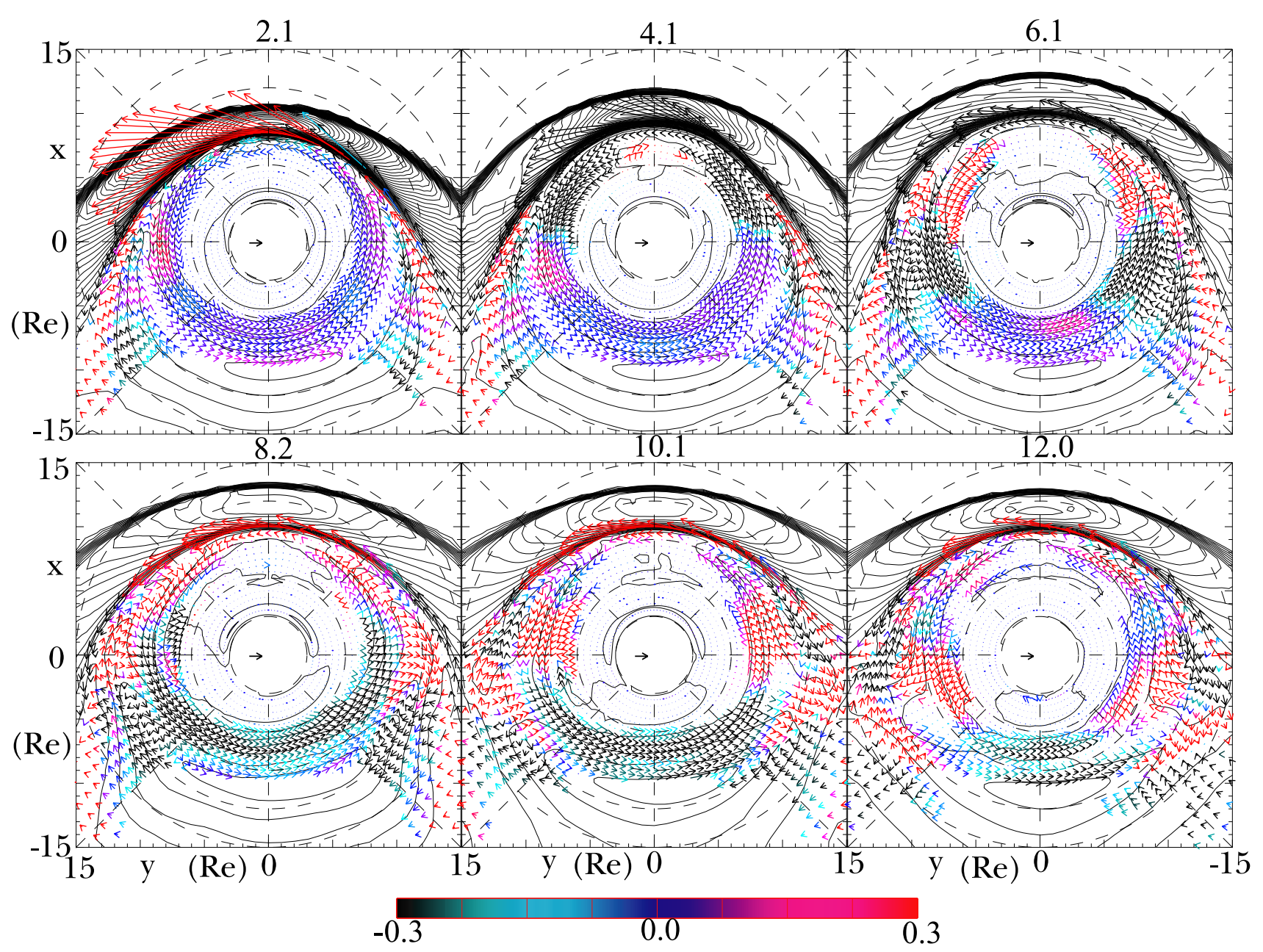

Fig. 4. Current vectors in the magnetospheric equatorial plane in the region of $|x|<15 R_{e}$ and $|y|<15 R_{e}$. The horizontal bar shown in the center of each panel indicates $J_{0}$. Color of arrows denotes $-J \cdot E$. Equi-contours of pressure are also shown with the contour interval of $1.8 p_{0}$. Vectors are drawn in the region where a magnetic field is almost perpendicular to the equatorial plane $\left(\left(B_{x}^{2}+B_{y}^{2}\right)^{1 / 2} / B_{z}<0.3\right)$. Number in the figure denotes time in minute.

\subsection{Magnetospheric current systems in the $\mathrm{SI}^{-}$period}

In order to confirm that the $\mathrm{SI}^{-}$has three phases like the $\mathrm{SI}^{+}$, we need to investigate the magnetospheric current system and the magnetospheric plasma flows. The mirror-image relationship is also investigated for the magnetospheric plasma processes. First, let us look at the current system. Figure 4 shows current vectors in the magnetospheric equatorial plane with equi-contours of plasma pressure from $2.1 \mathrm{~min}$ to $12.0 \mathrm{~min}$. Red and black colors of the current vectors indicate negative and positive $J \cdot E$. Note that negative/positive $J \cdot E$ means a dynamo/load for a current circuit.

At $4.1 \mathrm{~min}$, anti-sunward and outward (sunward and inward) currents are intensified in the post-noon (pre-noon) magnetosphere. These currents in the post- and pre-noon regions seem to be connected to each other via the nightside magnetospheric current. Since the dayside magnetopause current flows in the dawn-to-dusk direction, we do not observe a current vortex in the dayside magnetosphere unlike the case of the $\mathrm{SI}^{+}$with a counterclockwise current vortex (figure 3 of Fujita et al., 2003a). Therefore, this result seems that the PI current system in the magnetosphere does not have the mirror-image relationship between the $\mathrm{SI}^{-}$and the $\mathrm{SI}^{+}$. However, bearing in mind that a disturbed magnetospheric current excited by a solar wind impulse is superposed on a global-scale dawn-to-dusk current, we need to analyze only the disturbed part of the magnetospheric current. We roughly evaluate the disturbed current $(\delta J)$ as $J(4.1 \mathrm{~min})-J(0.0 \mathrm{~min})$ in Fig. 5. This figure shows that the disturbed magnetospheric current system draws a clockwise vortex in the dayside. Thus, spatial current pattern for the $\mathrm{SI}^{-}$is opposite to that of the $\mathrm{SI}^{+}$. This clockwise current vortex reduces magnetic field intensity. At the same time, plasma pressure is also reduced in the dayside magnetosphere. Therefore, simultaneous decreases in the magnetic field intensity and the pressure indicate that the disturbance a rarefactional wave of the compressional mode. A current generator of this current circuit is located in the magnetopause region because the dusk-to-dawn disturbed current in the magnetopause is anti-parallel to the dawn-to-dusk electric field excited by sudden expansion of the magnetosphere. Therefore, the $\mathrm{SI}^{-}$and the $\mathrm{SI}^{+}$have the current generators of the disturbed parts of the PI current system in the same region. 


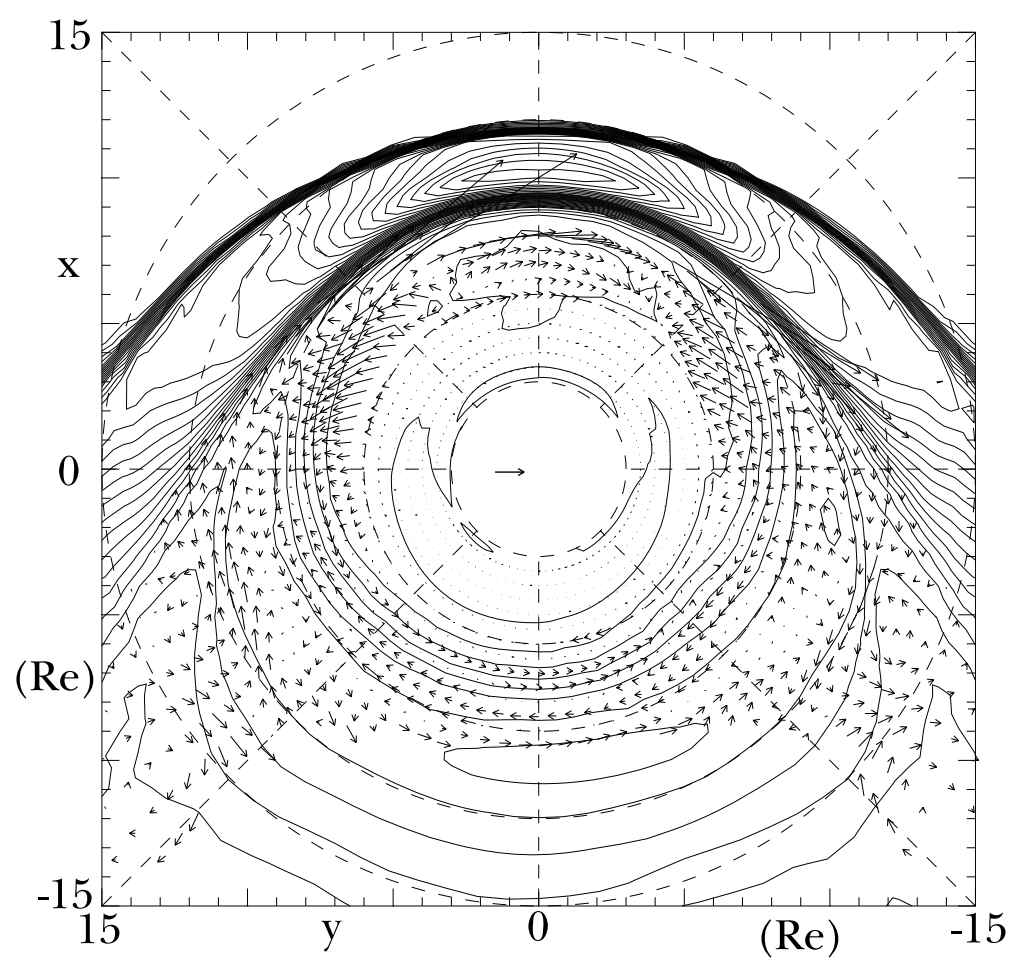

Fig. 5. Disturbed part of the current vectors in the magnetospheric equatorial plane at 4.1 min in the region of $|x|<15 R_{e}$ and $|y|<15 R_{e}$. The horizontal bar shown in the center of each panel indicates $0.5 J_{0}$. Equi-contours of pressure are also shown with the contour interval of $1.8 p_{0}$. Vectors are drawn in the region where a magnetic field at $0.0 \mathrm{~min}$ is almost perpendicular to the equatorial plane $\left(\left(B_{x}^{2}+B_{y}^{2}\right)^{1 / 2} / B_{z}<0.3\right)$ in the pre-SI ${ }^{-}$period.

Next, we consider the current system at $6.1 \mathrm{~min}$. Figure 4 shows negative $J \cdot E$ in the dayside inner magnetosphere. This region is located behind the wavefront of the rarefactional wave of the compressional mode. This situation is the same as the magnetospheric current of the first MI current system for the $\mathrm{SI}^{+}$(figure 10 of Fujita et al., 2003b). To investigate the magnetospheric current system at $6.1 \mathrm{~min}$, we draw a 3D picture of the current system in the top panel of Fig. 6. As a result, we confirm that the current system at 6.1 min is the first MI current system, but the current direction is opposed to that for the first MI current system of the $\mathrm{SI}^{+}$.

Last, at $8.2 \mathrm{~min}$, it is seemed from Fig. 3 that the Region 1 and 2 currents are recovered after extinction of the disturbances due to the negative solar wind impulse. However, we notice that there are enhanced FACs in the dayside ("C" $\mathrm{C}$ " of Fig. 3). The bottom panel of Fig. 6 shows that there are two magnetospheric current systems connecting the FACs of " $\mathrm{C}$ " in the dawn and dusk sides: (a) the current system via the dayside dawn-to-dusk magnetospheric current and (b) another in the nightside. We notice that the " $b$ " current system which carries FACs more than the "a" current is the Region 2 current system revealed by Tanaka (1995). Therefore, it is noteworthy that the current system in the second MI phase is essentially the Region 2 current in the case of the $\mathrm{SI}^{-}$instead of a modified Region 1 current in the case of the $\mathrm{SI}^{+}$. The "a" current system is driven associated with rebound of the magnetopause after rapid expansion of the magnetosphere. The rebound is equivalent with compression of the magnetosphere, which intensifies the magnetopause dawn-to-dusk current. Therefore, $J \cdot E<0$ in the magnetosphere. The re- bound will be explained later. It is noted that the " $a$ " current is peculiar to the $\mathrm{SI}^{-}$.

After $8.2 \mathrm{~min}$, the solar wind impulse, which passes over the magnetospheric flank, induces a localized depression of pressure (a pressure hole) in the nightside magnetosphere. However, this pressure hole is not so clear compared with the pressure island in the second MI phase of the $\mathrm{SI}^{+}$(Fujita et al., 2003b). Although not clear in Fig. 4, the anti-sunward propagation of the pressure hole, which modulates the magnetospheric current, results in anti-sunward shift of the Region 2 current peak location (Fig. 3).

\subsection{Magnetospheric plasma flow in the $\mathrm{SI}^{-}$period}

In the last part of this section, let us investigate the magnetospheric plasma flows in Fig. 7.

At $4.1 \mathrm{~min}$, the flows become sunward in the dayside, which means expansion of the dayside magnetosphere. This expansion stops at $6.1 \mathrm{~min}$. At $8.2 \mathrm{~min}$ and $10.1 \mathrm{~min}$, we observe anti-sunward flows in the outer dayside magnetosphere. This indicates rebound of the magnetosphere after the rapid expansion. Since the magnetosphere is compressed in the rebound phase, the electric current is driven in the magnetopause (Fujita et al., 2003a). Therefore, we confirm the rebound of the magnetosphere drives the "a" current in Fig. 6.

Figure 7 also shows the flow vectors with colors indicating the mode of the flow. Red and black vectors mean compressional and shear flows, respectively. Then, we recognize that the flows are compressional in the dayside magnetosphere at $4.1 \mathrm{~min}$. (Note that there is a rarefactional disturbance in the dayside magnetosphere.) The compressional disturbance 

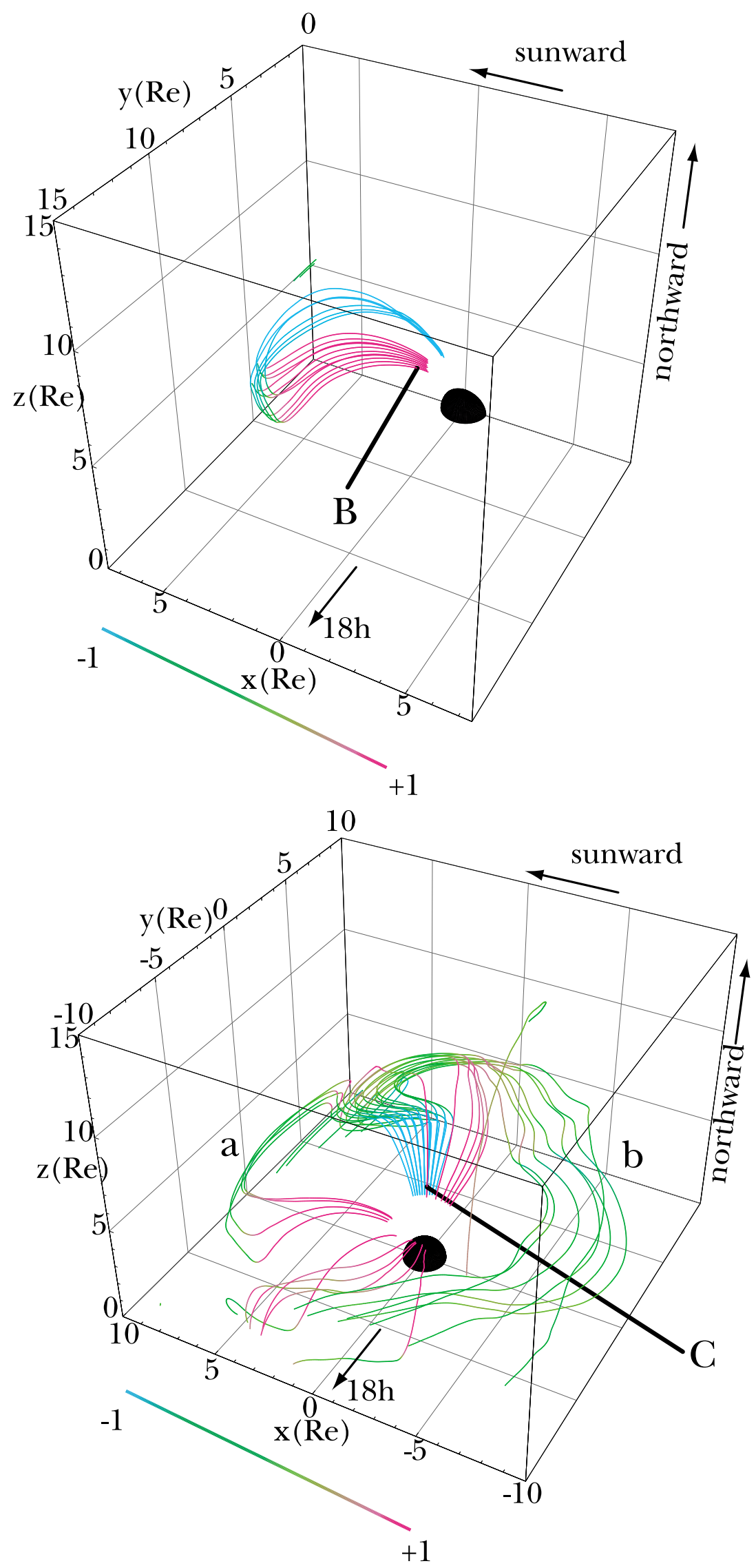

Fig. 6. 3D pictures illustrating (top) current lines downgoing to "B" in Fig. 3 at 6.1 min, and (bottom) those upgoing from " $C$ " in Fig. 3 at 8.2 min. Color of the curves denotes $\cos \theta$ where $\theta$ is the angle between a current and a magnetic field line. A red (blue) vector is parallel (anti-parallel) to the field line. The black sphere denotes the Earth in each panel. 


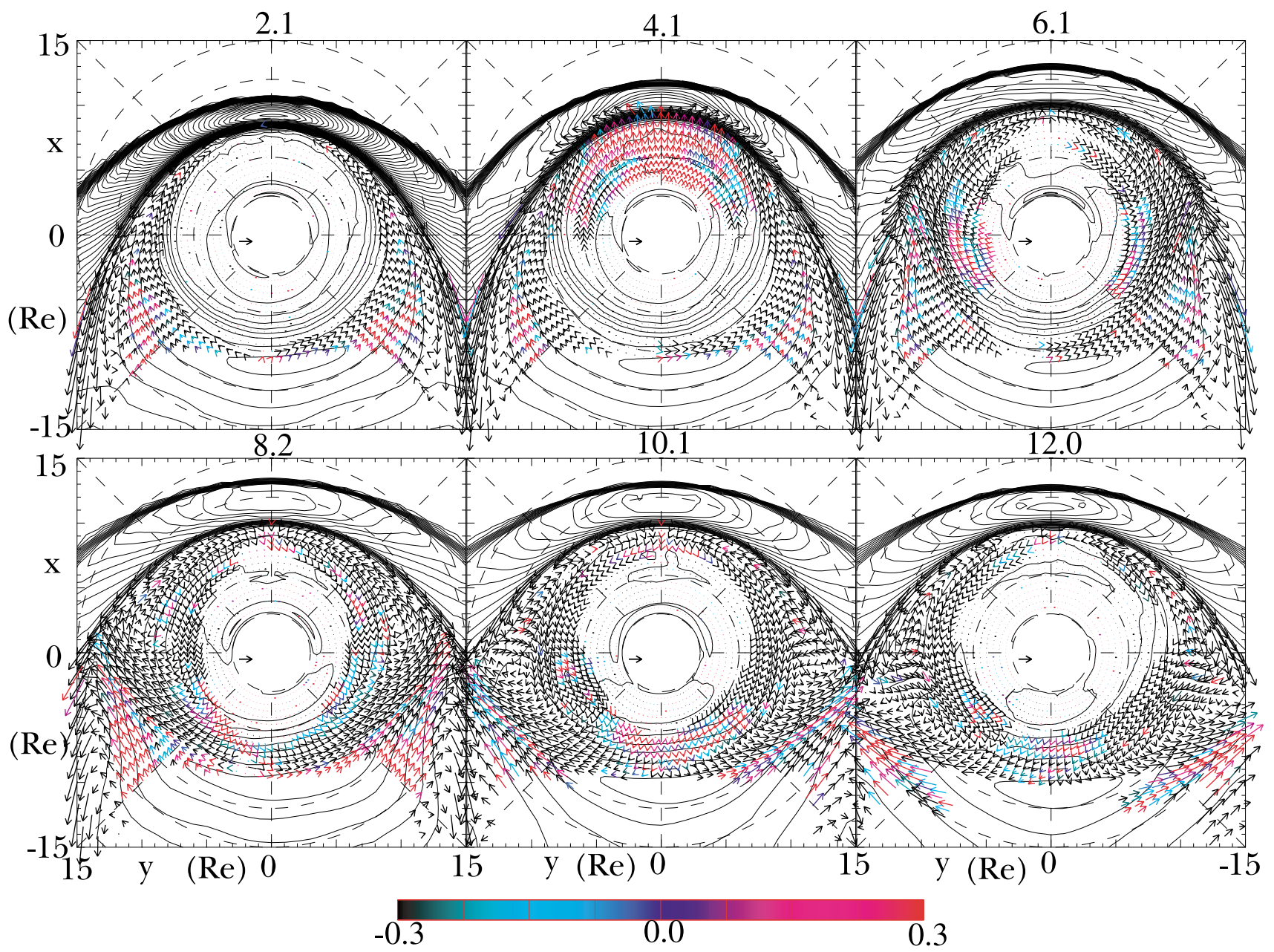

Fig. 7. Plasma flow vectors in the magnetospheric equatorial plane in the region of $|x|<15 R_{e}$ and $|y|<15 R_{e}$. Color of arrows denotes log $\left|\nabla v /(\nabla \times v)_{\|}\right|$ (red: compression, black: shear). Equi-contours of pressure are also shown with the contour interval of $1.8 p_{0}$. The horizontal bar shown in the center of each panel indicates $1.4 V_{0}$. Vectors are drawn in the region where a magnetic field is almost perpendicular to the equatorial plane $\left(\left(B_{x}^{2}+B_{y}^{2}\right)^{1 / 2} / B_{z}<0.3\right)$. Number in the figure denotes time in minute.

propagates in the anti-sunward direction at $6.1 \mathrm{~min}$. After 8.2 min, there are dominant shear flows in the magnetosphere except the outer nightside magnetosphere. These features of the flow mode in the $\mathrm{SI}^{-}$event is consistent with those of the $\mathrm{SI}^{+}$event.

Fujita et al. (2003b) found a transient convection vortex in the magnetosphere peculiar to the $\mathrm{SI}^{+}$in the second MI phase. This convection vortex is counterclockwise (clockwise) in the dusk (dawn) region. At the same time, the ionospheric potential is negative (positive) in the dusk (dawn) region. Fujita et al. (2003b) revealed that the magnetospheric convection flow vortex and the ionospheric flow vortex are consistent. This convection system is called as the $\mathrm{SC}$ transient cell convection. In the case of the $\mathrm{SI}^{-}$, Figure 7 shows the clockwise (counterclockwise) convection vortex in the dusk (dawn) region. This convection vortex shifts antisunward. In Fig. 2, we also see the positive (negative) potential in the dusk (a dawn) region. The peak of the potential also shifts anti-sunward. The magnetospheric convection is roughly corresponding to the ionospheric one. Therefore, the $\mathrm{SC}$ transient cell convection appears in the $\mathrm{SI}^{-}$event, too.

As noted above, there is a small-scale pressure depression (a pressure hole), although it is a little bit obscure. Therefore, the SC transient cell convection is related to a smallscale pressure variation in the magnetosphere in the $\mathrm{SI}^{-}$case, too. Finally, we conclude that plasma processes in the second MI phase are physically common between the $\mathrm{SI}^{-}$and the $\mathrm{SI}^{+}$except that the Region 2 and 1 currents play important roles in the $\mathrm{SI}^{-}$and in the $\mathrm{SI}^{+}$, respectively. Further physical implications of the SC transient cell convection will be discussed in another paper.

\section{Discussion}

Since Sato et al. (2001) reported sudden auroral brightening at the onset of the $\mathrm{SI}^{-}$, it is interesting to discuss a possible model of the sudden brightening as one of geophysical implications of the present numerical simulations.

It has been reported that sudden excitation and intensification of auroras, the cosmic noise absorption, and VLF waves occur at the onset of the SC $\left(\mathrm{SI}^{+}\right)$(Zhou and Tsurutani, 1999; Chua et al., 2001; Zhou et al., 2003; Hubert et al., 2003; Meurant et al., 2003). Zhou and Tsurutani (1999) demonstrated that auroral sudden brightening at the SC onset is caused by sudden compression of the dayside magne- 
tosphere that triggers particle precipitation due to enhanced pitch angle diffusion. On the other hand, Sato et al. (2001) reported that the auroral brightening occurs even in the $\mathrm{SI}^{-}$ event. This aurora is a discrete aurora associated with electron precipitation because simultaneous satellite observation detects "the inverted V" shape precipitation structure. They also discussed that repeated brightening of the auroras and synchronous variations of the geomagnetic variations imply quasi-periodic electron precipitation associated with a resonant ULF wave. This observation cannot be explained with a story based on the enhanced pitch angle diffusion because sudden expansion of the magnetosphere depresses the pitch angle diffusion efficiency.

Let us discuss a possible mechanism of sudden brightening of auroras at the onset of the $\mathrm{SI}^{-}$. FACs (the Alfvén mode) in the PI phase are excited through mode conversion from a compressional MHD wave (Tamao, 1964a, b, 1965; Araki, 1994; Lee and Hudson, 2001; Fujita et al., 2003a). The mode conversion process again plays a role in the case of $\mathrm{SI}^{-}$. Therefore, the Alfvén wave is excited in the PI phase. After Fujita et al. (2002), the field-line resonance appears almost simultaneously when the compressional MHD signal arrives at a region of non-uniform plasmas. (Although Fujita et al. (2002) considered $\mathrm{Pi} 2$ pulsations in the nightside magnetosphere, the theory can be applied to interaction between the compressional MHD wave due to solar wind impulse and the field-line resonance.) Namely, the field-line resonance is excited in the PI phase, although our MHD simulation cannot reproduce the field-line resonance correctly. Since Fujita et al. (2002) and the present MHD simulation cannot treat directly the electron precipitation that causes a discrete aurora, we need to consider another mechanisms beyond the MHD approximation. One candidate is the kinetic Alfvén wave (Hasegawa, 1976; Hasegawa and Chen, 1976; Lysak and Lotko, 1996) that has the field-aligned (parallel) electric field. When the field-line resonance wave has a perpendicular wavelength as small as the ion gyro-radius, the shear Alfvén wave becomes the kinetic Alfvén wave. Therefore, electrons are periodically accelerated by the parallel electric field of the kinetic Alfvén wave, which is consistent with the observation of Sato et al. (2001). In addition to this mechanism, the double layer (Goertz and Boswell, 1979; Sato and Okuda, 1981; Yajima and Machida, 2001) is another candidate of the mechanism for auroral particle acceleration. The double layer may accelerate again the electron that is first accelerated by the parallel electric field of the kinetic Alfvén wave. To confirm this model we need to carry out a 3D kinetic simulation in a realistic configuration. This is a future problem.

Next, we discuss observational confirmation of the abovementioned generation mechanism of the $\mathrm{SI}^{-}$-triggered auroras. First, we need to investigate the $\mathrm{SI}^{-}$-triggered auroras in the dawn region and in the dusk region. Our simulation, which shows an upward FAC in the dusk region, indicates that the $\mathrm{SI}^{-}$-triggered aurora should appear first in the dusk region. Further, since the field-line resonances in the dawn and dusk regions have $180^{\circ}$ phase difference, we expect alternative brightening of auroras in the dusk region and in the dawn region. In addition, the field-line resonance has an electric field symmetric with respect to the magneto- spheric equatorial plane, we observe upward FACs both in the northern and southern hemispheres simultaneously in the PI phase. Therefore, the repeated auroras after the onset of the $\mathrm{SI}^{-}$should be brightened synchronously at the conjugate points in the both hemispheres.

It may be interesting to compare the $\mathrm{SI}^{-}$-triggered aurora and the $\mathrm{SI}^{+}$-triggered aurora. In the latter case, auroral particle precipitation is possibly caused by both pitch angle diffusion due to sudden compression of the magnetosphere and the field-aligned acceleration of electrons. On the other hand, the pitch angle diffusion may be insignificant for the $\mathrm{SI}^{-}$-triggered aurora because sudden expansion of the magnetosphere is an unfavorable condition for enhanced pitch angle diffusion. Bearing in mind that particle precipitation due to the pitch angle diffusion excites a diffuse aurora, it is suggested that a discrete aurora and a diffuse aurora may be observed at the $\mathrm{SI}^{-}$onset and the $\mathrm{SI}^{+}$onset, respectively.

Note that the above discussions are just speculated. Further observations and theoretical considerations are needed for confirming this idea.

\section{Conclusion}

A geomagnetic negative sudden impulse $\left(\mathrm{SI}^{-}\right)$associated with a negative pressure impulse of the solar wind has been studied by using a solar wind-magnetosphere-ionosphere MHD model. We confirmed that the $\mathrm{SI}^{-}$is basically the mirror-image of the $\mathrm{SI}^{+}$. The other main results are as follows.

1) The plasma processes in the magnetosphere-ionosphere system are divided into a disturbed part due to the solar wind impulse and the background large-scale convection system in the PI phase and in the first MI phase. On the other hand, in the second MI phase, we cannot separate the two parts because the magnetospheric and ionospheric convection are self-consistently corresponding to each other.

2) The direction of the ionospheric FAC in the PI phase for the $\mathrm{SI}^{-}$is opposite to that for the $\mathrm{SI}^{+}$. Furthermore, the disturbed part of the magnetospheric current system for the $\mathrm{SI}^{-}$draws a clockwise current vortex in the equatorial plane, which is opposite to the counterclockwise current vortex in the case of the $\mathrm{SI}^{+}$.

3) The first MI current system of the $\mathrm{SI}^{-}$is a mirror-image of that in the $\mathrm{SI}^{+}$case.

4) In the second MI phase, the $\mathrm{SI}^{-}$also has the SC transient cell convection. The rotation sense of the convection is reversed between the $\mathrm{SI}^{-}$and the $\mathrm{SI}^{+}$.

5) $\mathrm{The} \mathrm{SI}^{-}$-triggered auroras may be explained by a model where the kinetic Alfvén wave is excited in association with the field-line resonance converted from the compressional wave in the PI phase.

Acknowledgments. The authors are very grateful for discussions with T. Araki, S. Machida and T. Takeuchi of Kyoto University and N. Sato of National Institute of Polar Research. They also thank the anonymous reviewers for useful comments. Numerical calculations were performed by NEC SX6 installed at the Communications Research Laboratory and the Computer Center of the National Insti- 
tute of Polar Research, as well as Computer Center of Nagoya University. S. F. expresses his gratitude to T. Ogino of Solar Terrestrial Environment Laboratories for utilization of the Nagoya University Computer and H. Fujita of Univ. Tokyo for arrangement of personal computers used for graphical presentations. This work was partly supported by the Japan Society for the Promotion of Science (JSPS) under Grant-in-Aid for Scientific Research 14540415.

\section{References}

Araki, T., A physical model of the geomagnetic sudden commencement, in Solar Wind Sources of Magnetospheric Ultra-Low-Frequency Waves, edited by M. J. Engebretson, K. Takahashi, and M. Scholer, pp. 183-200, American Geophysical Union, Washington, D.C., 1994.

Araki, T. and H. Nagano, Geomagnetic response to sudden expansions of the magnetosphere, J. Geophys. Res., 93, 3983-3988, 1988.

Chua, D., G. Parks, M. Brittnacher, W. Peria, G. Germany, J. Spann, and C. Carlson, Energy characteristics of auroral electron precipitation: A comparison of substorms and pressure related auroral activity, J. Geophys. Res., 106, 5945-5956, 2001.

Fujita, S., M. Itonaga, A. Yoshikawa, H. Nakata, and T. Mizuta, A numerical simulation of the Pi2 pulsations associated with the substorm current wedge, J. Geophys. Res., 107(A3), 10.1029/2001JA000137, 2002.

Fujita, S., T. Tanaka, T. Kikuchi, K. Fujimoto, K. Hosokawa, and M. Itonaga, A numerical simulation of the geomagnetic sudden commencement: 1. Generation of the field-aligned current associated with the preliminary impulse, J. Geophys. Res., 108(A12), 1416, doi:10.1029/2002JA009407, 2003a.

Fujita, S., T. Tanaka, T. Kikuchi, K. Fujimoto, and M. Itonaga, A numerical simulation of the geomagnetic sudden commencement: 2. Plasma processes in the main impulse, J. Geophys. Res., 108(A12), 1417, doi:10.1029/2002JA009763, 2003b.

Goertz, C. K. and R. W. Boswell, Magnetosphere-ionosphere coupling, J. Geophys. Res., 84, 7239-7246, 1979.

Hasegawa, A., Particle acceleration by MHD surface wave and formation of aurora, J. Geophys. Res., 81, 5083-5090, 1976.

Hasegawa, A. and L. Chen, Kinetic processes in plasma heating by resonant mode conversion of Alfvén wave, Phys. Fluids, 19, 1924-1934, 1976.

Hubert, B., J. C. Gérard, S. A. Fuselier, and S. B. Mende, Observation of dayside subauroral proton flashes with the IMAGE-FUV imagers, Geophys. Res. Lett., 30(3), 1145, doi:10.1029/2002GL016464, 2003.

Iijima, T. and T. A. Potemra, Field-aligned currents in the dayside cusp observed by Triad, J. Geophys. Res., 81, 5971-5979, 1976.

Keller, K. A., M. Hesse, M. Kuznetsova, L. Rastätter, T. Moretto, T. I. Gombosi, and D. L. DeZeeuw, Global MHD modeling of the impact of a solar wind pressure change, J. Geophys. Res., 107(A7), 10.1029/2001JA000060, 2002

Lee, D.-H. and M. L. Hudson, Numerical studies on the propagation of sudden impulses in the dipole magnetosphere. J. Geophys. Res., 106, 8435-8445, 2001.

Lysak, R. L. and W. Lotko, On the kinetic dispersion relation for shear Alfvén waves, J. Geophys. Res., 101, 5085-5094, 1996.

Meurant, M., J.-C. Gérard, B. Hubert, V. Coumans, C. Blockx, N. Østgaard, and S. B. Mende, Dynamics of global scale electron and proton precipitation induced by a solar wind pressure pulse, Geophys. Res. Lett., 30(20),
2032, doi:10.1029/2003GL018017, 2003.

Sato, T. and H. Okuda, Numerical simulations on ion acoustic double layers, J. Geophys. Res., 86, 3357-3368, 1981.

Sato, N., Y. Murata, H. Yamagishi, A. S. Yukimatsu, M. Kikuchi, M. Watanabe, K. Makita, H. Yang, R. Liu, and F. J. Rich, Enhancement of optical aurora triggered by the solar wind negative impulse $\left(\mathrm{SI}^{-}\right)$, Geophys. Res. Lett., 28, 127-130, 2001.

Slinker, S. P., J. A. Fedder, W. J. Hughes, and J. G. Lyon, Response of the ionosphere to a density pulse in the solar wind: Simulation of travelling convection vortices, Geophys. Res. Lett., 26, 3,549-3,552, 1999.

Takeuchi, T., T. Araki, H. Lühr, O. Rasmussenn, J. Watermann, D. K. Milling, I. R. Mann, K. Yumoto, K. Shiokawa, and T. Nagai, Geomagnetic negative sudden impulse due to a magnetic cloud observed on May 13, 1995, J. Geophys. Res., 105, 18,835-18,846, 2000.

Takeuchi, T., T. Araki, A. Viljanen, and J. Watermann, Geomagnetic negative sudden impulses: Interplanetary causes and polarization distribution, J. Geophys. Res., 107(A07), 10.1029/2001JA900152, 2002.

Tamao, T., The structure of three-dimensional hydromagnetic waves in a uniform cold plasma, J. Geomag. Geoelectr., 16, 89-114, 1964a.

Tamao, T., A hydromagnetic interpretation of geomagnetic SSC*, Rep. Ionos. Space Res. Japan, 18, 16-31, 1964b.

Tamao, T., Transmission and coupling resonance of hydromagnetic disturbances in the non-uniform Earth's magnetosphere, Sci. Rep. Tohoku Univ., Series 5, Geophysics, 17, 43-72, 1965.

Tanaka, T., Finite Volume TVD Scheme on an unstructured grid system for three-dimensional MHD simulation of inhomegeneous systems including strong background potential fields, J. Compt. Phys., 111, 381-389, 1994.

Tanaka, T., Generation mechanisms for magnetosphere-ionosphere current systems deduced from a three-dimensional MHD simulation of the solar wind-magnetosphere-ionosphere coupling processes, J. Geophys. Res., 100, 12057-12074, 1995.

Tanaka, T., Configuration of the magnetosphere-ionosphere convection system under northward IMF conditions with nonzero IMF $\mathrm{B}_{y}, J$. Geophys. Res., 104, 14683-14690, 1999.

Tanaka, T., The state transition model of the substorm onset, J. Geophys. Res., 105, 21081-21096, 2000a.

Tanaka, T., Field-aligned current systems in the numerically simulated magnetosphere, in Magnetospheric Current Systems, edited by S.-I. Ohtani, R. Fujii, M. Hesse, and R. L. Lysak, p. 53, American Geophysical Union, Washington, D.C., $2000 \mathrm{~b}$.

Yajima, A. and S. Machida, Electrostatic particle simulations of the WDL in the auroral plasma including the effects of up-flowing ions, Earth Planets Space, 53, 139-147, 2001

Zhou, X. and B. T. Tsurutani, Rapid intensification and propagation of the dayside aurora: Large scale interplanetary pressure pulses (fast shock), Geophys. Res. Lett., 26, 1097-1100, 1999.

Zhou, X.-Y., R. J. Strangeway, P. C. Anderson, D. G. Sibeck, B. T. Tsurutani, G. Haerendel, H. U. Frey, and J. K. Arballo, Shock aurora: FAST and DMSP observations, J. Geophys. Res., 108(A4), 8019, doi:10.1029/2002JA009701, 2003.

S. Fujita (e-mail: sfujita@mc-jma.ac.jp), T. Tanaka, T. Kikuchi, and S. Tsunomura 